\title{
Parametric identification algorithm for asynchronous electric motor inverter on the switching intervals of power transistors
}

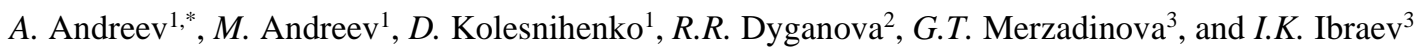 \\ ${ }^{1}$ Vologda State University, Vologda, Russia \\ ${ }^{2}$ Kazan State Power Engineering University, Kazan, Russia \\ ${ }^{3}$ L.N. Gumilyov Eurasian National University, Astana, Kazakhstan
}

\begin{abstract}
The authors propose an algorithm for identifying the parameters of a controlled asynchronous electric drive in real time, which provides calculation of stator and rotor resistances which change as a function of temperature. The algorithm is based on the analysis of a current tube of electric motor phase with the subsequent calculation of resistances.
\end{abstract}

\section{Introduction}

In most cases, when solving problems of identifying the parameters of an asynchronous electric drive, the timeaveraged values of the observed variables are taken into account, regardless of the method of its identification [14]. The obtained values, being the initial ones for calculating the parameters, provide controllability within the framework of linearized models of electric drive, despite the apparent discreteness and nonlinearity of the system. The transition to identification, taking into account the instantaneous values of the observed variables, makes it possible to identify, at discrete time intervals, areas of linear change of variables, at which the drive system is linear.

The discrete time intervals in modern electric drives with vector control are uniquely related to the pulsewidth modulation frequencies and the switching frequencies of the power switches. The duration of the key switching intervals lies in the range of tens (maximum of hundreds) of microseconds, which clearly makes it possible to consider the system linear in the small. The temperature drift of active resistances of rotor and stator of asynchronous motor is usually not taken into account when solving control problems, and if it is taken into account, the method is in building thermal models implemented as separate software modules [58]. Next, an algorithm for indirect determination of stator active resistance in real time is considered.

\section{Theoretical part}

In the proposed concept of identification, the classical model in the coordinate system fixed relative to the stator, the mechanical component of which is characterized by the inertia moment and the resistance moment given to the motor shaft, is adopted as the basic mathematical model of an asynchronous motor.

The shaft rotation speed changes insignificantly at the switching intervals due to their short duration. Therefore the third equation of system (1) loses its meaning and may not be considered. Next, we perform a series of transformations and obtain an expression for the time constant $T$ :

$$
\left\{\begin{array}{l}
u_{s}(t)=R_{s} \times i_{s}(t)+L_{s} \times \frac{d i_{s}(t)}{d t}+L_{m} \times \frac{d i_{r}(t)}{d t}, \\
0=R_{r} \times i_{r}(t)+L_{m} \times \frac{d i_{s}(t)}{d t}+L_{r} \times \frac{d i_{r}(t)}{d t}-j \omega(t) \times L_{m} \times i_{s}(t)-j \omega(t) \times L_{r} \times i_{r}(t), \\
J(t) \times \frac{d \omega(t)}{d t} \times \frac{1}{p_{\tau}}=\frac{3}{2} \times p_{\tau} \times L_{m} \times I_{m}\left(\overline{i_{s}}(t) \times i_{r}(t)\right)-M_{c}(t),
\end{array}\right.
$$

where $R_{s}, R_{r}$ are winding resistances of stator and rotor, $L_{s}, L_{r}, L_{m}$ are winding inductances of stator and rotor, and magnetizing inductance, $p_{\tau}$ is amount of pole pairs, $J(t)$ is inertia moment, $M_{c}(t)$ is resistance moment, $u_{s}(t)$ is supply voltage, $i_{s}(t), i_{r}(t)$ are currents of stator and rotor.

\footnotetext{
*Corresponding author: alexu79@mail.ru
} 


$$
\begin{aligned}
& T=2 \times R_{r} \times R_{S} \times\left(L_{r} \times R_{s}+L_{s} \times R_{r}\right) \cdot 2 \times \omega^{2} \times L_{r} \times \\
& \times R_{s} \times\left(L_{m}^{2} \cdot L_{r} \times L_{s}\right) /\left(\left(R_{r} \times R_{s}\right)^{2}+\left(\omega \times L_{r} \times R_{s}\right)^{2}\right)
\end{aligned}
$$

The resulting expression is valid for considering the system at the switching intervals of the power switches of the autonomous inverter. It can be used during synthesis of the speed control loop for high-precision AC drives, where the angular rotation velocity is calculated or measured.

The transfer coefficient $K$ and the time constant $T$ for $\mathrm{DC}$ is calculated as follows

$$
K=\frac{1}{R_{E Q}}, T=\frac{L_{E Q}}{R_{E Q}}=L_{E Q} \Rightarrow L_{E Q}=\frac{T}{K}
$$

That is

$$
\frac{\left|U_{S Q}\right|}{\left|I_{S Q}\right|}=\left(\frac{1}{k^{2}}+\left(\omega_{c} \times \frac{T}{K}\right)^{2}\right)^{1 / 2}
$$

where $\omega_{c}=2 \pi f, f=50 \mathrm{~Hz}$.

Further, using the experimental curve of the "tube" of current, the qualitative view of which, together with the modulated stator phase voltage, is shown in Figure 1, it is necessary to solve the problem of determining the time constant $T$ on the switching intervals of the power switches of an autonomous inverter. This task is reduced to solving a single linear differential equation for a DC circuit with the following values known: voltage, two samples of time (duration of the switching interval) and current at the beginning and end of the switching interval.

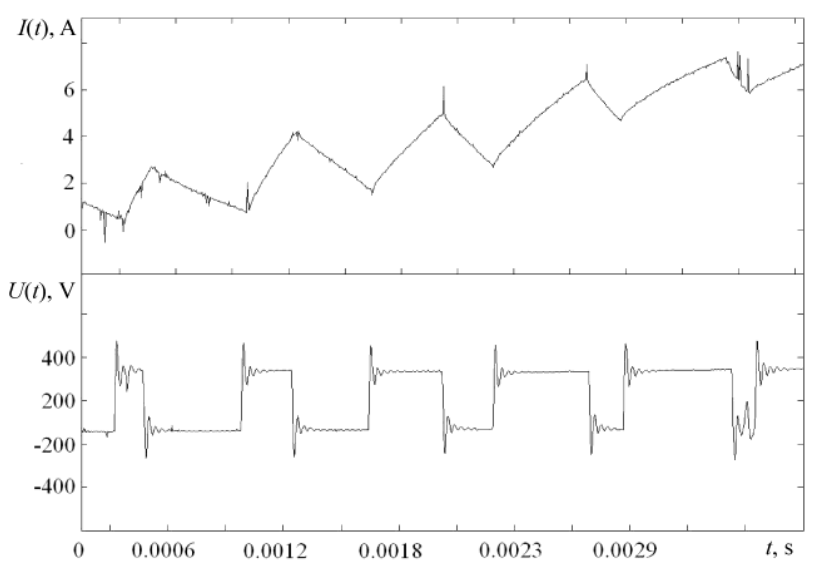

Fig. 1. Experimental curve of current tube and modulated voltage.

After a series of assumptions and mathematical transformations, we obtain the following expression [5]:

$$
\left\{\begin{array}{l}
\frac{\left|U_{S Q}\right|}{\left|I_{S Q}\right|}=\left(\frac{1}{k^{2}}+\left(\omega_{c} \times \frac{T}{K}\right)^{2}\right)^{1 / 2} \\
T=t \cdot\left(\ln \left(\frac{I_{o}-K \times U}{I(t)-K \times U}\right)\right)^{-1}
\end{array}\right.
$$

where $t$ is duration of the switching interval; $I_{0}$ is current at the beginning of the switching interval; $I(t)$ is current at the end of the switching interval; $U$ is DC voltage during the switching interval.

The information contained in the current tube is sufficient to identify the time-varying parameters of the electric drive by appropriate calculations.

The main nuance is that the resulting expression (5), which is a solution of a linear inhomogeneous differential equation, allows one to determine $T$ only in the absence of time gaps. The current tube is a piecewise-exponential function that is discontinuous in time.

$$
T=t \cdot\left(\ln \left(\frac{I_{o}-K \times U}{I(t)-K \times U}\right)\right)^{-1}
$$

To obtain reliable results, it is necessary to preliminarily restore a piecewise exponential function in order to obtain continuous exponential function, which is used to determine the time constant $T$. The essence of the restoration method is shown in Figure 2

Figure 2 shows a fragment of the current tube at the growing segment of the quasi-sinusoidal current of the stator phase. The fragment corresponds to ten switching intervals of power switches of the autonomous inverter. We take into account only those areas where the keys providing the increasing phase current are open. The essence of the restoration is to find the same values of the increasing current in the neighboring areas of exponent. The intervals between the time stamps, where the currents in adjacent areas are the same, are cut out. Thus, the continuous exponential function is restored, which is necessary to determine $T$ from expression (5).

The proposed method of the exponent restoration is called the splicing method;

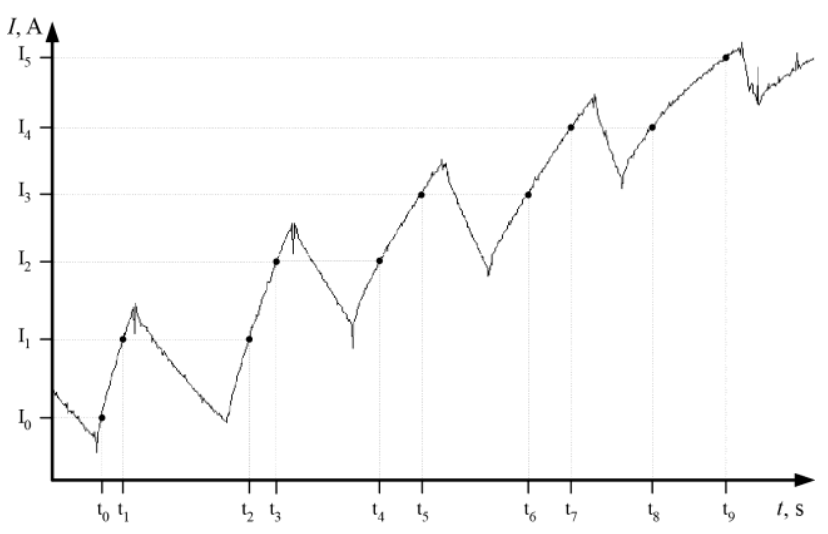

Fig. 2. Exponent restoration. 
The most rational option for implementing a realtime identifier which uses the instantaneous values of stator currents and switched phase voltage of motor is creation of a resident program module. The algorithm of the module is based on a list of computational procedures in accordance with the developed methodology.

As in the regular resident identifiers of modern frequency converters, preliminary identification is carried out using classical approaches. The results of this identification are measured and calculated resistances and inductances of equivalent circuit used in the drive control system of the electric drive.

The sequence of operations of the algorithm of the proposed identifier consists in carrying out procedures of both preliminary and real-time identification with data transfer to the control system.

\subsection{Preliminary identification}

1. During preliminary identification mode, a constant voltage is applied to two phases of the asynchronous motor. According to the established values of current and voltage, the stator resistance of the cold engine $R_{s x}$ is calculated using the formula

$$
R_{s x}=\frac{U_{S T}}{I_{S T}}
$$

The transmission coefficient $K$ is determined using the expression

$$
K=\frac{I_{S T}}{U_{S T}}=\frac{1}{R_{S X}}
$$

2. The motor is started at the rated speed with a load close to the rated one using a frequency converter. In the steady state mode, after 2-3 seconds of work, until the engine temperature has changed, the instantaneous values of currents and voltages of any of the stator phases are recorded and saved. If the computing resources of the control system are sufficient, it is possible to use the same data for all phases of the stator.

3 . Restoration of the exponent from the stored values of the current tube of the stator phase in accordance with explanations to Figure 1.

4. Calculation of time constant $T$ and equivalent inductance $L e$ from the restored exponent using the expressions

$$
\begin{aligned}
& T=\left(t_{E N D}-t_{B E G}\right)\left(\ln \frac{I_{E N D}-K \times U_{S T}}{I_{B E G}-K \times U_{S T}}\right)^{-1}, \\
& L_{E Q}=T \times R_{S X}
\end{aligned}
$$

5. Save the $L_{E Q}$ value as a constant for subsequent calculations.

6. Transfer of $L_{E Q}, R_{s x}, T$ values through the information channel to the control system.

The results of preliminary identification of the stator active resistance for a cold engine are necessary to determine the equivalent inductance of the motor phase, which is further considered an "absolute" constant. Since the preliminary identification was used to determine the resistance of the two phases of the stator, and the current tube was obtained for one phase, in further calculations we use the half-resistance value $R_{S}$.

\subsection{Real-time identification}

1. Fixing and preservation the instantaneous values of currents and voltages of any of the stator phases at the growing segment of the phase current quasi-sinusoid.

2. Restoration of the exponent from the stored values of current tube of the stator phase in accordance with the explanations to Figure 1.

3. Calculation of the theoretical steady-state current value $I_{S T}$ of the stator phase by solving an extrapolation problem.

4. Calculation of coefficient $K$ and time constant $T$ with known Le, $I_{S T}, U_{S T}$ in accordance with the expressions.

$$
\begin{gathered}
K=\frac{I_{S T}}{U_{S T}} \\
T=\left(t_{E N D}-t_{B E G}\right)\left(\ln \frac{I_{E N D}-K \times U_{S T}}{I_{B E G}-K \times U_{S T}}\right)^{-1}
\end{gathered}
$$

5. Calculation of current stator phase resistance using two expressions.

$$
R_{s r}^{1}=\frac{1}{K}, R_{s r}^{2}=\frac{L_{E Q}}{T}
$$

6. Checking the coincidence of the calculated values $R_{s r}^{1}$ and $R_{s r}^{2}$ with a predetermined accuracy (in the case of a significant residual, repeat the execution of paragraph 2).

7. Calculation of the average value of $R_{s r}$ when meeting the requirements of accuracy and transfer of the calculated values of $R_{s r}$ and $T$ to the level of the control system.

\section{Experimental part}

To confirm the obtained results, an experimental stand [6] was assembled on the basis of the Department of Control and Computing Systems of Vologda State University, the structure of which is shown in Figure 3.

The frequency converter (FC) Omron Varispeed F7 is used to control the rotation speed of an asynchronous motor with a short-circuit rotor. The PC has the CXDrive software necessary for controlling the frequency converter. On the motor shaft there is an electromagnetic powder brake, which performs the function of the load unit and allows one to create a passive braking torque. The brake control system allows one to adjust the braking torque both manually and automatically using a PC. On the other side of the shaft a pulse speed sensor is mounted. The signal produced by the sensor is fed to the 


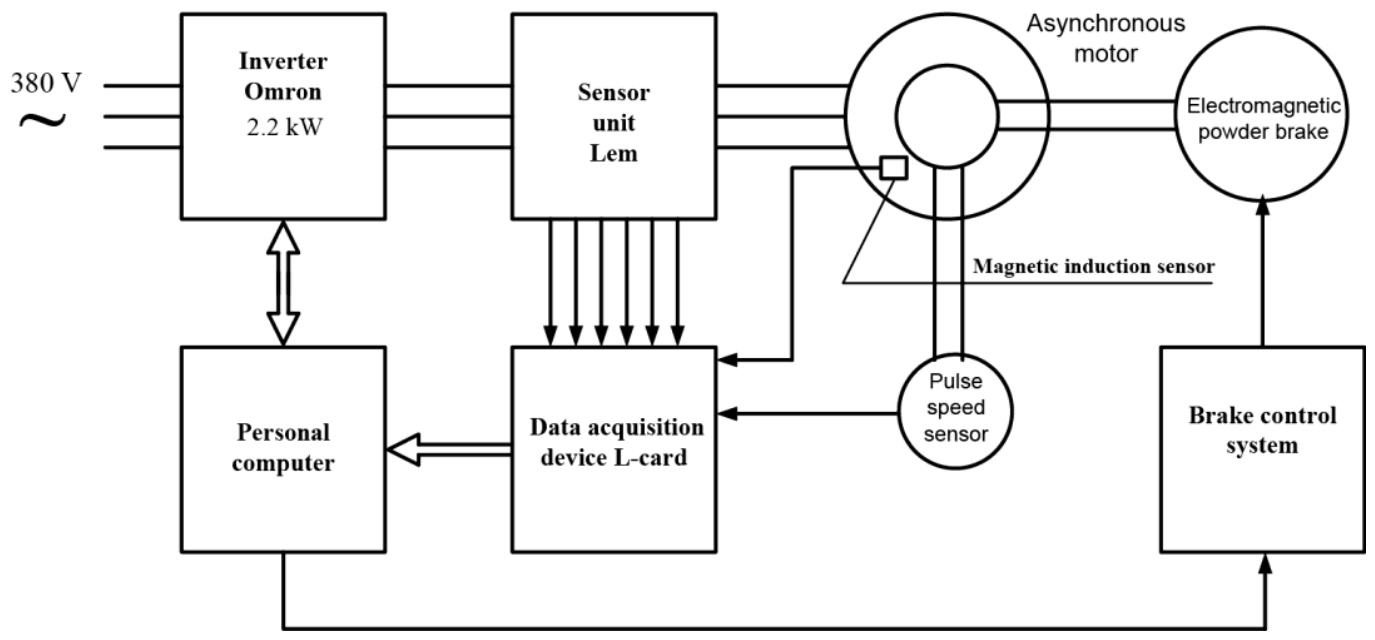

Fig. 3. Structure of the complex.

input of the electrical signal analysis system. Computer analysis of electrical signals consists of two parts. The first group consists of current, voltage and magnetic induction sensors (optional), which convert the measured values into normalized signals for a data acquisition device (multi-channel L-Card L-783 ADC). The second part is the data acquisition device itself, which transfers the received data via the PCI interface to the computer. LabView 8, CX-Drive, LGraph software is installed on the PC. It provides ample opportunities for analyzing and processing data from the data acquisition device board, which allows one to control the frequency converter. At the same time, LabView 8 allows one to work with external devices in order to generate control actions for the electromagnetic powder brake and frequency converter. The functionality of the research complex provides a test drive in static and dynamic modes of operation. The appearance of the stand is shown in Figure 4.

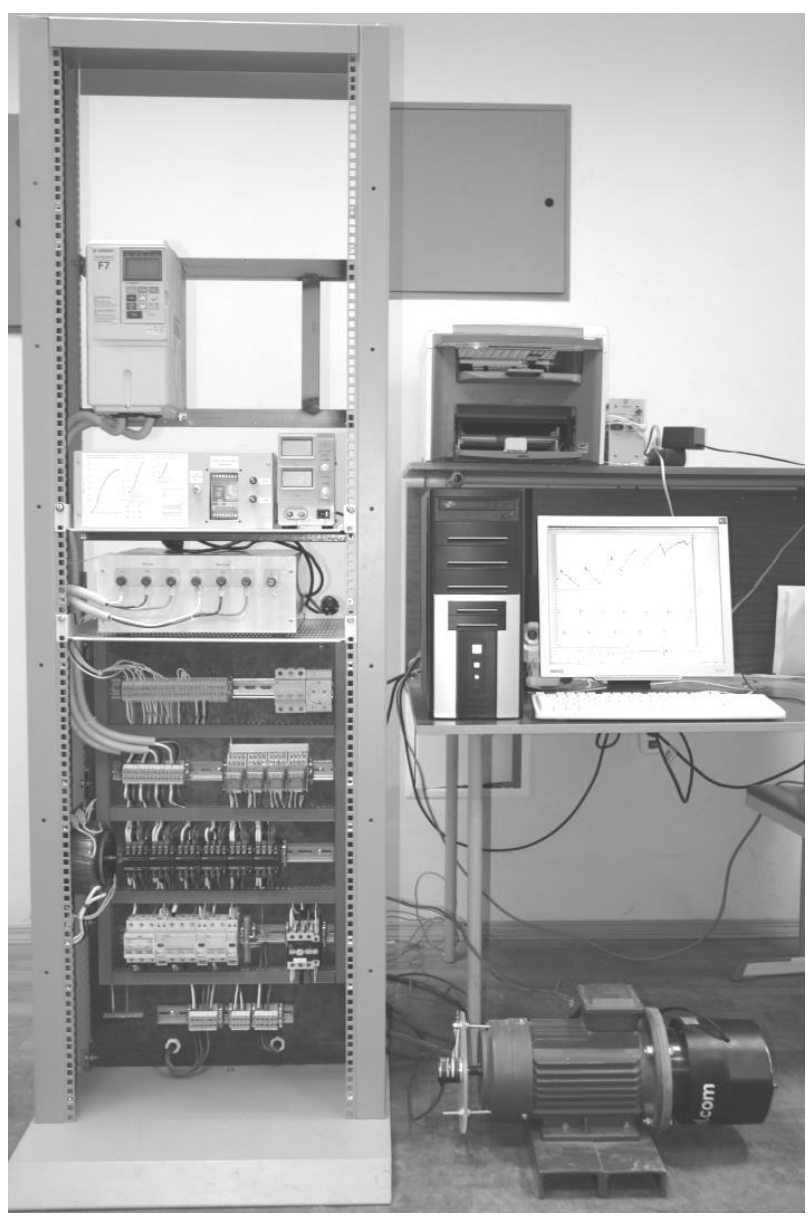

Fig. 4. Appearance of the test stand.

The test program includes preliminary identification and real-time identification.

\subsection{Preliminary identification}

1. Measurement of the interface resistance by technical means of the CIMR-F7 frequency converter and CX-Drive software at various temperatures of the motor housing surface. 
2. Recording data of current transient when the two stator phases are supplied with a constant stabilized voltage at various temperatures of the motor housing surface.

\subsection{Real-time identification}

Registration of the current tube at various modulation frequencies, rotation speeds, loads and temperatures measured on the surface of the motor housing. During registration the following values vary:

PWM frequency: from 2 to $15 \mathrm{kHz}$;

Motor rotation speed: from 300 to $3000 \mathrm{rpm}$;

Load: from idle to nominal;

Temperature: 22 to $70^{\circ} \mathrm{C}$.

Below is an example of the obtained oscillogram of the stator current when a constant voltage is applied in 2 phases.

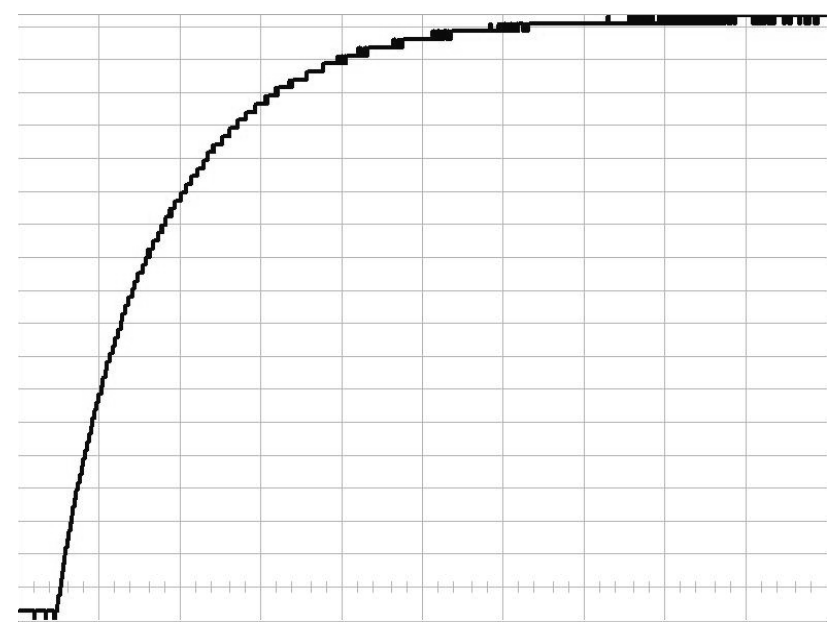

Fig. 5. Transient when applying DC voltage to the stator.

In accordance with the experimental data and computational procedures of the identification algorithm $[8,9]$, the ratios of direct measurements and calculations are presented. The discrepancy between the measured and the calculated resistances of the stator windings does not exceed $5 \%$. The insignificant discrepancy is due to insufficient accuracy of fixing the temperatures of the object in the modes of measurement and identification. Temperatures were measured on the motor surface, the thermal conductivity of motor materials was not taken into account, the error in measuring the temperature was $\pm 1^{\circ} \mathrm{C}$. The temperature discrepancy in identical experiments was $\pm 5^{\circ} \mathrm{C}$.

\section{Conclusions}

The proposed identification algorithm in real time can be most effectively used in precision asynchronous electric drives, where the accuracy of the control variables of the drive variables in both static and dynamic modes of operation depends on the accuracy of computational algorithms.

\section{References}

[1] M.G. Popov, D.E. Petrushin, N.S. Efimov, Increasing the emergency control systems efficiency in Kola's and Karelia's power systems (Proceedings of the 2019 IEEE Conference of Russian Young Researchers in Electrical and Electronic Engineering, ElConRus 2019) 1040-1043 (2019)

[2] E.N. Desyatirikova, A.V. Vasilenko, A.V. Polukazakov, V.E. Mager, L.V. Chernenkaya, Structural synthesis of a dynamic system control algorithm based on the separation of electromagnetic and electromechanical movements, (Proceedings of the 2019 IEEE Conference of Russian Young Researchers in Electrical and Electronic Engineering, ElConRus 2019) 471-473 (2019)

[3] I. Boguslawsky, N. Korovkin, M. Hayakawa, A.C. Large, Machines: Theory and investigation methods of currents and losses in stator and rotor meshes including operation with nonlinear loads (Book), 550 (2016)

[4] V. Vanin, A. Bulychov, M. Popov, O. Vasilyeva, M. Shakhova, Measurement of currents and voltages non-sinusoidal parameters in power supply systems with rectifier load (MATEC Web of Conferences, 245) (2018)

[5] M.A. Andreev, A.N. Andreev, A.M. Vodovozov, A.S. Elyukov, Information Technologies for Modeling and Management 4(56), 588-595 (2009)

[6] A.N. Andreev, D.A. Kolesnichenko, N.M. Kolesnichenko, Measurement Techniques 61(8), 812 816 (2018)

[7] D.A. Kolesnichenko, A.N. Andreev, Russian Metallurgy (Metally) 12, 1025-1032 (2017)

[8] M.A. Andreev, A.N. Andreev, A.M. Vodovozov, A.S. Elyukov, Automation and energy saving of machine-building and metallurgical production, technology and reliability of machines, devices and equipment: Materials of the fifth international scientific and technical conference 1, 30-33 (2009)

[9] M.A. Andreev, A.N. Andreev, A.M. Vodovozov, A.S. Elyukov, Automation and energy saving of machine-building and metallurgical production, technology and reliability of machines, devices and equipment: Materials of the fifth international scientific and technical conference 1, 34-38 (2009) 